ON THE X-RAY SPECTRA OF ANOMALOUS X-RAY PULSARS AND SOFT GAMMA REPEATERS

This article has been downloaded from IOPscience. Please scroll down to see the full text article.

2010 ApJ 710 L115

(http://iopscience.iop.org/2041-8205/710/2/L115)

The Table of Contents and more related content is available

Download details:

IP Address: 131.215.193.213

The article was downloaded on 26/03/2010 at 21:26

Please note that terms and conditions apply. 


\title{
ON THE X-RAY SPECTRA OF ANOMALOUS X-RAY PULSARS AND SOFT GAMMA REPEATERS
}

\author{
V. M. KASPI ${ }^{1,2,3}$ AND K. Boydstun ${ }^{1,2}$ \\ ${ }^{1}$ Department of Physics, Rutherford Physics Building, McGill University, 3600 University Street, Montreal, Quebec, H3A 2T8, Canada; vkaspi@ physics.mcgill.ca \\ ${ }^{2}$ Department of Astronomy, California Institute of Technology, Pasadena, CA 91125, USA \\ Received 2009 August 10; accepted 2010 January 14; published 2010 January 29
}

\begin{abstract}
We revisit the apparent correlation between soft X-ray band photon index and spin-down rate $\dot{v}$ previously reported for Anomalous X-ray Pulsars (AXPs) and Soft Gamma Repeaters (SGRs) by Marsden \& White. Our analysis, improved thanks to new source discoveries, better spectral parameter measurements in previously known sources, and the requirement of source quiescence for parameter inclusion, shows evidence for the previously noted trend, although with greater scatter. This trend supports the twisted magnetosphere model of magnetars although the scatter suggests that factors other than $\dot{v}$ are also important. We also note possible correlations involving the spectra of AXPs and SGRs in the hard X-ray band. Specifically, the hard-band photon index shows a possible correlation with inferred $\dot{v}$ and $B$, as does the degree of spectral turnover. If the former trend is correct, then the hard-band photon index for AXP 1E 1048.1 - 5937 should be $\sim 0-1$. This may be testable with long integrations by the International Gamma-Ray Astrophysics Laboratory, or by the upcoming focusing hard X-ray mission NuSTAR.
\end{abstract}

Key words: pulsars: general - stars: magnetic field - stars: neutron - X-rays: stars

\section{INTRODUCTION}

Anomalous X-ray Pulsars (AXPs) were long thought of as "soft" X-ray sources, because their X-ray spectra below $10 \mathrm{keV}$ were observed to be falling off sharply. International GammaRay Astrophysics Laboratory (INTEGRAL) and Rossi X-ray Timing Explorer ( $R X T E$ ) observations of these objects, as well as of their close cousins, the Soft Gamma Repeaters (SGRs), revealed that their spectra turn over in the 10-20 keV range, such that in fact some AXPs are the hardest known sources in the sky in the hard X-ray band (Molkov et al. 2004; Kuiper et al. 2004; Mereghetti et al. 2005; den Hartog et al. 2006; Götz et al. 2006). Here we define the "soft" X-ray band as $1-10 \mathrm{keV}$, and the "hard" X-ray band as $20-80 \mathrm{keV}$.

Prior to the discovery of the hard X-ray emission, Marsden \& White (2001, hereafter MW01) reported an apparent correlation between soft-band spectral hardness and spin-down rate $\dot{v}$, where $v$ is the spin frequency. This reported correlation has been used as the primary motivation for the "twisted magnetosphere" model for magnetars (Thompson et al. 2002; Thompson \& Beloborodov 2005; Beloborodov \& Thompson 2007). In that model, surface activity driven by the decay of the large internal magnetic field of the star results in a twisted dipolar morphology of the magnetosphere, with powerful currents emanating from the surface and subsequently returning to heat it. In this picture, the MW01 hardness/ $\dot{v}$ correlation is interpreted physically as a correlation between hardness and the spin-down-inferred surface dipolar magnetic field $B$, where the latter can be estimated via $B \equiv 3.2 \times 10^{19}(P \dot{P})^{1 / 2} \mathrm{G}$, with $P \equiv 1 / v$ being the pulse period. The simple $\dot{v} / \mathrm{B}$ connection exists because, for AXPs and SGRs, there is a far wider range in observed $\dot{v}$ than observed $v$. In the twisted magnetosphere model, this correlation is a result of stronger currents, due to higher $B$ hence higher activity, resonantly upscattering seed thermal photons from the hot surface to form the nonthermal component. In addition to the apparent soft-band hardness $/ B$ correlation reported by MW01, support for this picture comes from observed correlations between soft-band spectral index and flux in individual variable

\footnotetext{
3 Lorne Trottier Chair; Canada Research Chair.
}

sources (e.g., Tiengo et al. 2005; Rea et al. 2005; Campana et al. 2007; Tam et al. 2008), since all else being equal, higher fluxes are expected from larger twists, because of higher return currents.

In this Letter, we revisit the correlation reported by MW01 in light of three main developments in the field since their work. First, several new sources have been discovered since 2001 and can be used to confirm the original claimed correlation, which was based on only seven objects. Second, previously known sources have been observed in longer integrations with higher sensitivity instruments, allowing better determinations of their spectral parameters. Third, since 2001, it has been recognized that AXPs can exhibit great variability both in the soft X-ray flux and spectrum, particularly during outbursts (e.g., Kaspi et al. 2003; Woods et al. 2004; Israel et al. 2007; Tam et al. 2008). However, MW01 considered measured magnetar spectral parameters without concern for the objects' outburst status at the time of measurement, thereby possibly using data from objects in very different spectral states.

In addition, with the study of the hard-band emission from AXPs and SGRs well underway, there now exist enough data for correlations similar to that noted by MW01 to be considered. Indeed, Götz et al. (2006) noted qualitatively that in the hard band, AXPs are harder than SGRs, the reverse of what is seen in the soft band. Here we consider this point in more detail, noting possible correlations between the hardness of the hardband spectra of AXPs and SGRs and $B$ (and $\dot{v}$ ), and between magnitude of spectral change from the soft to hard bands, and $B$ (and $\dot{v}$ ).

\section{COMPILING AXP AND SGR SPECTRA}

We characterize AXP and SGR soft and hard X-ray spectra phenomenologically. Although significant effort has been expended to do physically motivated broadband spectral modeling (e.g., Lyutikov \& Gavriil 2006; Rea et al. 2007a; Özel \& Guver 2007; Nobili et al. 2008; Zane et al. 2009), this work is still progressing, with no final word reached. Moreover, our motivation here is to characterize all sources in a systematic and uniform way. Hence we choose to parameterize soft-band 
Table 1

Spectral Parameters for Non-transient SGRs and AXPs ${ }^{\mathrm{a}}$

\begin{tabular}{|c|c|c|c|c|c|c|c|c|c|}
\hline Source Name & $\begin{array}{c}B \\
\left(10^{14} \mathrm{G}\right)\end{array}$ & $\begin{array}{c}\dot{v} \\
\left(10^{-12} \mathrm{~s}^{-2}\right)\end{array}$ & $\begin{array}{r}\mathrm{BB} k T \\
(\mathrm{keV})\end{array}$ & $\Gamma_{s}$ & $\begin{array}{l}\text { Reference } \\
\text { for } \Gamma_{s}, k T\end{array}$ & $\Gamma_{h}^{p}$ & $\begin{array}{c}\text { Reference } \\
\text { for } \Gamma_{h}^{p}\end{array}$ & $\Gamma_{h}^{t}$ & $\begin{array}{c}\text { Reference } \\
\text { for } \Gamma_{h}^{t}\end{array}$ \\
\hline 1E $2259+586$ & 0.59 & -0.0099 & $0.411(4)$ & $4.10(3)$ & 1 & $-1.02_{0.13}^{0.24}$ & 12 & $\ldots$ & $\ldots$ \\
\hline CXO J164710.2 - 455216 & $\sim 0.9^{\mathrm{b}}$ & $-0.007^{\mathrm{b}}$ & $0.49(10)^{\mathrm{c}}$ & $3.5_{0.3}^{1.3 \mathrm{c}}$ & 2 & $\ldots$ & $\ldots$ & $\ldots$ & $\cdots$ \\
\hline $4 \mathrm{U} 0142+61$ & 1.3 & -0.026 & $0.386(5)^{\mathrm{c}}$ & $3.67(9)^{\mathrm{c}}$ & 3 & $0.40(15)$ & 13 & $0.93(6)$ & 13 \\
\hline $1 \mathrm{E} 1547.0-5408$ & 2.2 & -5.41 & $0.43_{0.04}^{0.03 \mathrm{c}}$ & $3.7_{2.0}^{0.8 \mathrm{c}}$ & 4 & $\cdots$ & $\cdots$ & $\cdots$ & $\cdots$ \\
\hline CXOU J010043.1 - 721134 & 3.9 & -0.29 & $0.35(6)^{\mathrm{c}}$ & $1.8(1)^{\mathrm{c}}$ & 5 & $\ldots$ & $\ldots$ & $\ldots$ & $\ldots$ \\
\hline $1 \mathrm{E} 1048.1-5937$ & 4.2 & -0.65 & $0.56(1)$ & $3.14(11)$ & 6 & $\ldots$ & $\ldots$ & $\ldots$ & $\ldots$ \\
\hline 1RXS J170849.0 - 400910 & 4.7 & -0.16 & $0.456_{0.007}^{0.004 c}$ & $2.792_{0.012}^{0.008 c}$ & 7 & $1.01(12)$ & 12 & $1.44(45)$ & 12 \\
\hline SGR 1900+14 & 6.4 & -2.91 & $0.47(2)^{\mathrm{c}}$ & $1.9(1)^{\mathrm{c}}$ & 8 & $\ldots$ & $\ldots$ & $3.1(5)$ & 14 \\
\hline $1 \mathrm{E} 1841-045$ & 7.1 & -0.30 & $0.44(2)$ & $2.0(3)$ & 9 & $0.72(15)$ & 12 & $1.32(11)$ & 12 \\
\hline SGR $0526-66$ & 7.3 & -1.00 & $0.48(5)$ & $3.12(8)$ & 10 & $\ldots$ & $\ldots$ & $\ldots$ & $\ldots$ \\
\hline SGR $1806-20$ & 21 & -9.62 & $0.476(39)^{\mathrm{d}}$ & $1.67(15)$ & 11 & $\ldots$ & $\ldots$ & $2.0(2)^{\mathrm{c}}$ & 15 \\
\hline
\end{tabular}

Notes.

${ }^{a}$ Numbers in parentheses represent $1 \sigma$ uncertainties in the least significant digit(s) unless otherwise indicated (see c).

${ }^{\mathrm{b}} B$ and $\dot{v}$ for this source are not yet measured precisely; see Woods et al. (2009).

$\mathrm{c}$ These uncertainties represent $90 \%$ confidence intervals.

$\mathrm{d}$ The BB temperature is from a different observation as the reported $\Gamma_{s}$; see Woods et al. (2007).

References. (1) Woods et al. 2004; (2) Israel et al. 2007; (3) White et al. 1996; (4) Gelfand \& Gaensler 2007; (5) McGarry et al. 2005; (6) Tam et al. 2008; (7) Rea et al. 2007b; (8) Mereghetti et al. 2006; (9) Morii et al. 2003; (10) Kulkarni et al. 2003; (11) Woods et al. 2007; (12) Kuiper et al. 2006; (13) den Hartog et al. 2008b; (14) Götz et al. 2006; (15) Esposito et al. 2007.

spectra using the simple blackbody plus power-law model, with $\Gamma_{s}$ being the soft-band photon index (defined as $d N / d E \propto$ $\left.E^{-\Gamma_{s}}\right)$. Similarly, we parameterize the hard-band spectrum by a power law of photon index $\Gamma_{h}$ (defined as for $\Gamma_{s}$ ). We recognize that this parameterization is flawed, both in the soft band where the true spectrum is likely to be better represented by a Comptonized thermal model (such as those currently under development) as required in the twisted magnetosphere model, but also more dramatically in the mid-energy range where there is clearly spectral curvature. Nevertheless, the parameterization is convenient, used ubiquitously in the literature, and does a reasonable job of characterizing the soft- and hard-band spectra currently available, given statistical uncertainties (see, e.g., Kuiper et al. 2006). We further acknowledge that the spectra, both soft (e.g., Patel et al. 2001) and hard (e.g., den Hartog et al. 2008a), are dependent on pulse phase; however, here we consider only phase-averaged spectral parameters.

In Table 1, we summarize the results reported in the literature for all known non-transient magnetars for which there are such measurements. Since MW01, several new magnetars have been discovered, four of which have well-measured spin and spectral parameters (CXOU J010043.1 - 721134, SGR 0526 - 66, 1E 1547.0 - 5408, and CXO J164710.2 - 455216). In addition, in the soft X-ray band, SGRs and AXPs are now established to be highly variable, such that $\Gamma_{s}$ can vary greatly. In compiling Table 1, we took great care to include the $\Gamma_{s}$ (always for a blackbody plus power-law model) for objects when their reported fluxes were at or near the lowest yet recorded. This was done to ensure the objects were in their quiescent state, with no temporary enhancement that could be interpreted as a magnetospheric twist with corresponding spectral hardening. By contrast, MW01 averaged together different published values of $\Gamma_{s}$, presumably under the assumption that the values do not change, which we now know to be incorrect. In making our selections from the literature, where multiple quiescent spectral observations were available, we verified that the reported $\Gamma_{s}$ values were roughly consistent, then selected that with the smallest reported uncertainty. We excluded the so-called transient AXP XTE J1810 - 197 which shows no clear nonthermal component when in quiescence (Gotthelf et al. 2004).

\section{SOFT-BAND SPECTRAL INDICES}

Using the data provided in Table 1 , we plot $\Gamma_{s}$ versus $B$ and $\dot{v}$ in Figure 1. Values of $\dot{v}$ are long-term averages as recorded in the SGR/AXP online catalog ${ }^{4}$ (see references for $\dot{v}$ values therein). We note the previously seen trend for decreasing $\Gamma_{s}$ with increasing $B$ or $\dot{v}$. To quantify this trend, we determined Pearson's linear correlation coefficient to be $r=-0.56$ for a sample size of $N=11$, corresponding to a null-hypothesis (two-tailed) probability of $p=0.076$. We note that this $p$ value, as for all others in this Letter, is actually an upper limit given that uncertainties are not accounted for in calculating $r$. This $\Gamma_{s} / \dot{v}$ trend is not as striking as it was in MW01 (compare with their Figure 2); there is more scatter. ${ }^{5}$ If we ignore AXP $1 \mathrm{E}$ $1547.0-5408$, whose $\Gamma_{s}$ has large uncertainty, the trend is improved and is significant with $>99 \%$ confidence $(r=-0.78$ for $N=10, p=0.0079$ ), though still with considerable scatter. Interestingly, the plot of $\Gamma_{s}$ versus $B$ (Figure 1, left) shows a better correlation $(r=-0.82$ for $N=11, p=0.0022)$, which may support the twisted magnetosphere model in that it is the magnitude of $B$ that determines the twist size, not just the spin-down rate. Nevertheless, there is scatter; this could imply that the value of $B$, as inferred from spin-down parameters, is only a rough estimate of the true field strength, a possibility already generally recognized given the greatly simplified assumptions in computing $B$, and also observationally suggested from the absence of anomalous X-ray emission from very high- $B$ rotation-powered pulsars (RPPs; e.g., Pivovaroff et al. 2000; McLaughlin et al. 2003; Kaspi \& McLaughlin 2005).

We also searched for a correlation between the simultaneously measured blackbody temperature $k T$ and $B$ (see Figure 2), but, like MW01, found none. Specifically, for $N=11, r=$ 0.29 , implying $p=0.38$, clearly non-excluding of the null hypothesis. This argues that the underlying physical parameter that is most closely linked with a magnetar's magnetic field is manifested in the power-law component, rather than in the thermal component (see also Kulkarni et al. 2003).

\footnotetext{
4 www.physics.mcgill.ca/ pulsar/magnetar/main.html

5 We estimate for MW01's reported $\Gamma_{s}$ vs. $\dot{v}$ correlation (their Figure 2), $r=-0.83$ for $N=7$, implying $p=0.021$.
} 

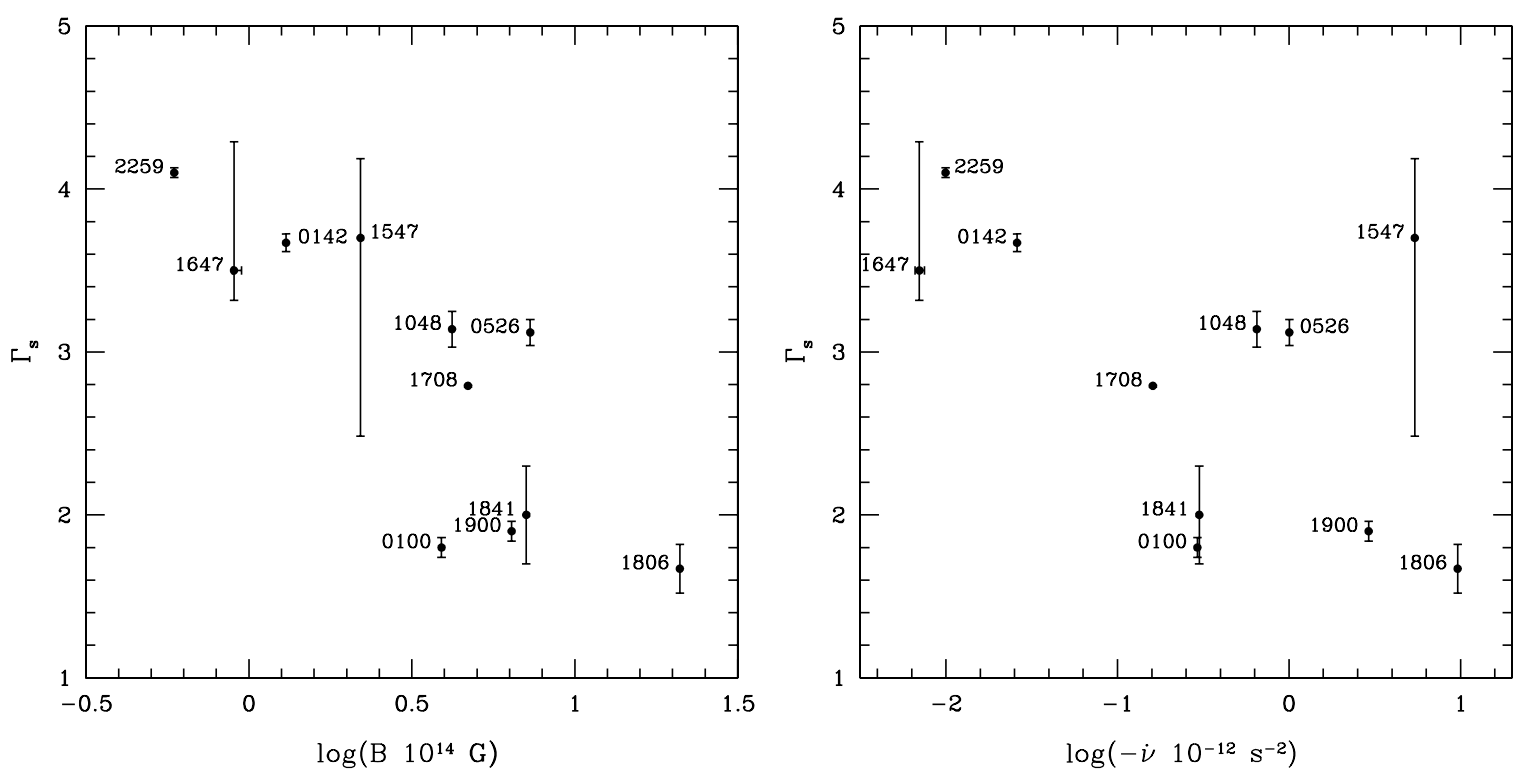

Figure 1. Left: $\Gamma_{s}$ vs. B. See the text and Table 1 for data used and references. Right: same but vs. $\dot{v}$. Both: error bars represent $1 \sigma$ uncertainties.

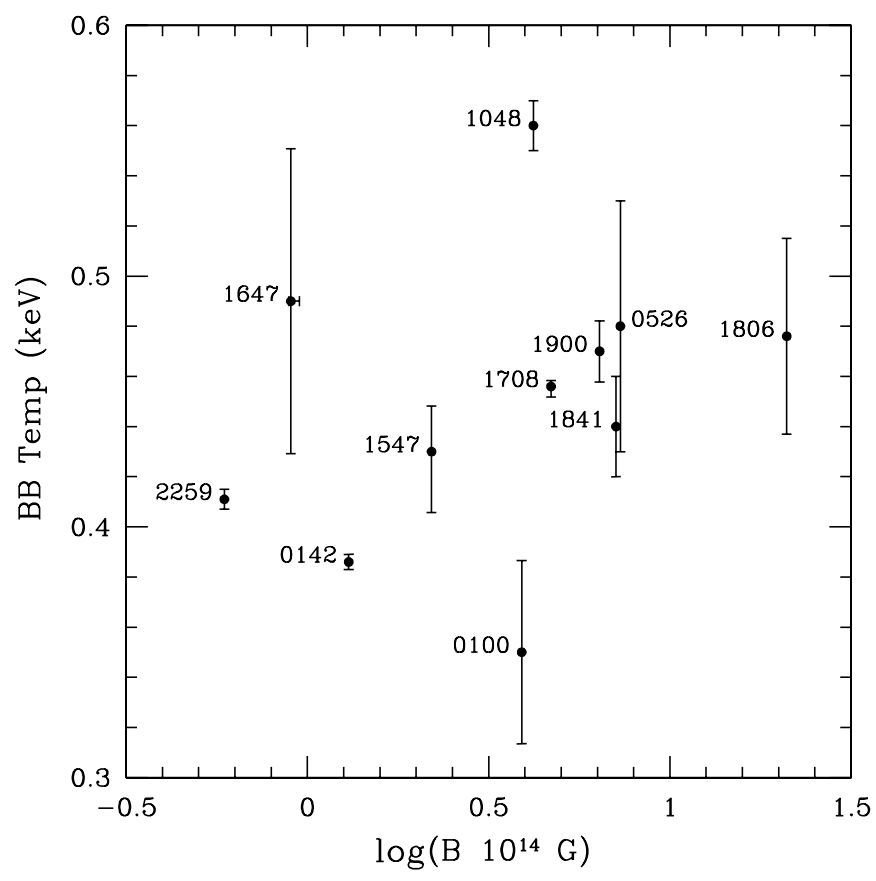

Figure 2. Blackbody temperature vs. $B$, for all magnetars for which $\Gamma_{s}$ is measured. See the text and Table 1 for data used and references. Error bars represent $1 \sigma$ uncertainties.

\section{HARD-BAND SPECTRAL INDICES}

Figure 3 shows values of $\Gamma_{h}$ plotted versus $B$ (left) and $\dot{v}$ (right) for the six sources for which $\Gamma_{h}$ has presently been measured. In some cases, $\Gamma_{h}$ is for the pulsed emission, in other cases for the total emission, and in three cases for both (see Table 1). There is a possible trend in the data. AXPs are harder in the $20-80 \mathrm{keV}$ band than are SGRs as qualitatively noted by Götz et al. (2006). But as is clear here, even within the AXP class itself, low $B$ or $\dot{v}$ sources are harder than high $B$ or $\dot{v}$ sources. To be more quantitative, for the sources for which the pulsed emission is measured, we find $r=0.88$ for $N=4$, implying $p=0.12$ for a $\Gamma_{h}$ correlation with $\log B$ (Figure 3, left), and $r=0.86$ for $N=4$ implying $p=0.14$ for the
$\Gamma_{h}$ versus $\log \dot{v}$ data (Figure 3, right). These values are more significant for the total flux $(r=0.79, N=6, p=0.062$ and $r=0.84, N=6, p=0.034$ for $\log B$ and $\log \dot{v}$, respectively) when the (pulsed) value for 1E 2259+586 is included, under the assumption that its total flux $\Gamma_{h}$ does not differ much from its pulsed $\Gamma_{h}$, as for the three sources for which both are measured. Although not quite significant at the $>99 \%$ confidence level, these possible correlations seem noteworthy.

We note that the value of $\Gamma_{h}$ for $1 \mathrm{E} 2259+586$ should be regarded with caution, the source having been detected only up to $30 \mathrm{keV}$ thus far. This will hopefully improve with time. We also of course caution that the trend is defined by only six sources, one fewer than was used by MW01. If this correlation is correct, then for AXP 1E 1048.1 - 5937, the brightest AXP for which $\Gamma_{h}$ has not yet been measured, $\Gamma_{h}$ should be $\sim 0-1$. This may be testable with long integrations by INTEGRAL, or by the future focusing hard X-ray mission NuSTAR (expected launch 2011) which should have point-source sensitivity of $\sim 100$ times that of INTEGRAL.

Note that in all but two cases (4U 0142+61 and SGR $1806-$ 20 ), the sources were in quiescence at all epochs when the hard X-ray observations were done. For $4 \mathrm{U} 0142+61$, the measured hard X-ray spectral parameters are an average over $\sim 4$ years of data (den Hartog et al. 2008b) which included a few-month active period in 2006 (Gavriil et al. 2009). This active period seems unlikely to have biased the hard X-ray spectrum because of the absence of soft X-ray variability during that epoch (Gavriil et al. 2009), and because of the relatively small duration of the hard X-ray exposure during the active period relative to during quiescence, as well as because of the absence of any evidence for hard X-ray flux or spectral variability over the four years of INTEGRAL observations (with flux and $\Gamma_{h}$ stable to within 17\%; den Hartog et al. 2008b). For SGR 1806 - 20, the hard $\mathrm{X}$-ray observations were obtained roughly two years after the source's giant flare of 2004 December. During the hard X-ray observations, the source was nearly back to quiescence, though still bursting occasionally. Whether this mild activity affected its hard X-ray spectrum is unknown given that no variability has yet been reported in the hard X-ray spectrum of any magnetar. This will likely be difficult to determine with current hard 

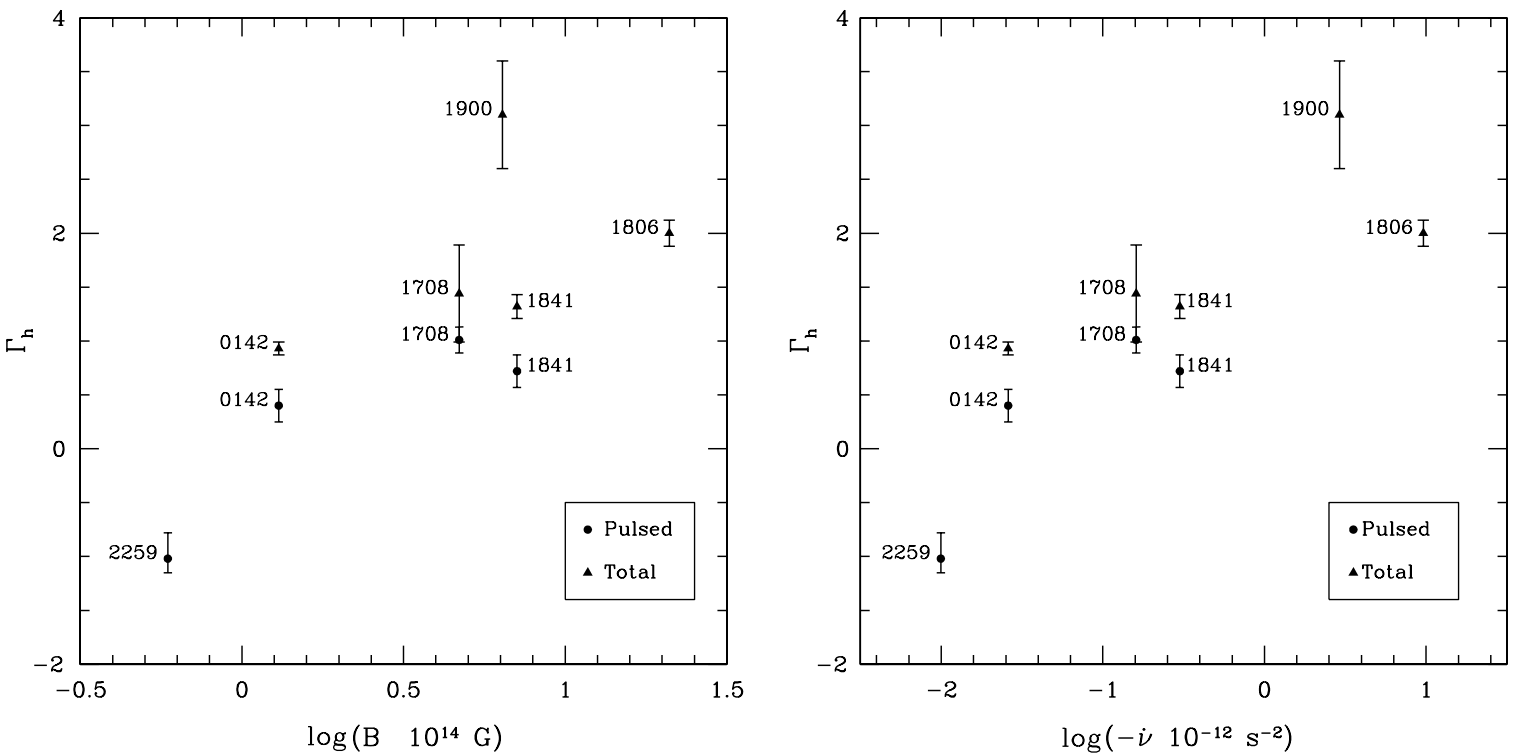

Figure 3. Left: $\Gamma_{h}$ vs. $B$, for all magnetars for which $\Gamma_{h}$ is measured. See the text and Table 1 for data used and references. Circles represent pulsed flux, and triangles represent total flux. Right: same but vs. $\dot{v}$. Both: error bars represent $1 \sigma$ uncertainties.
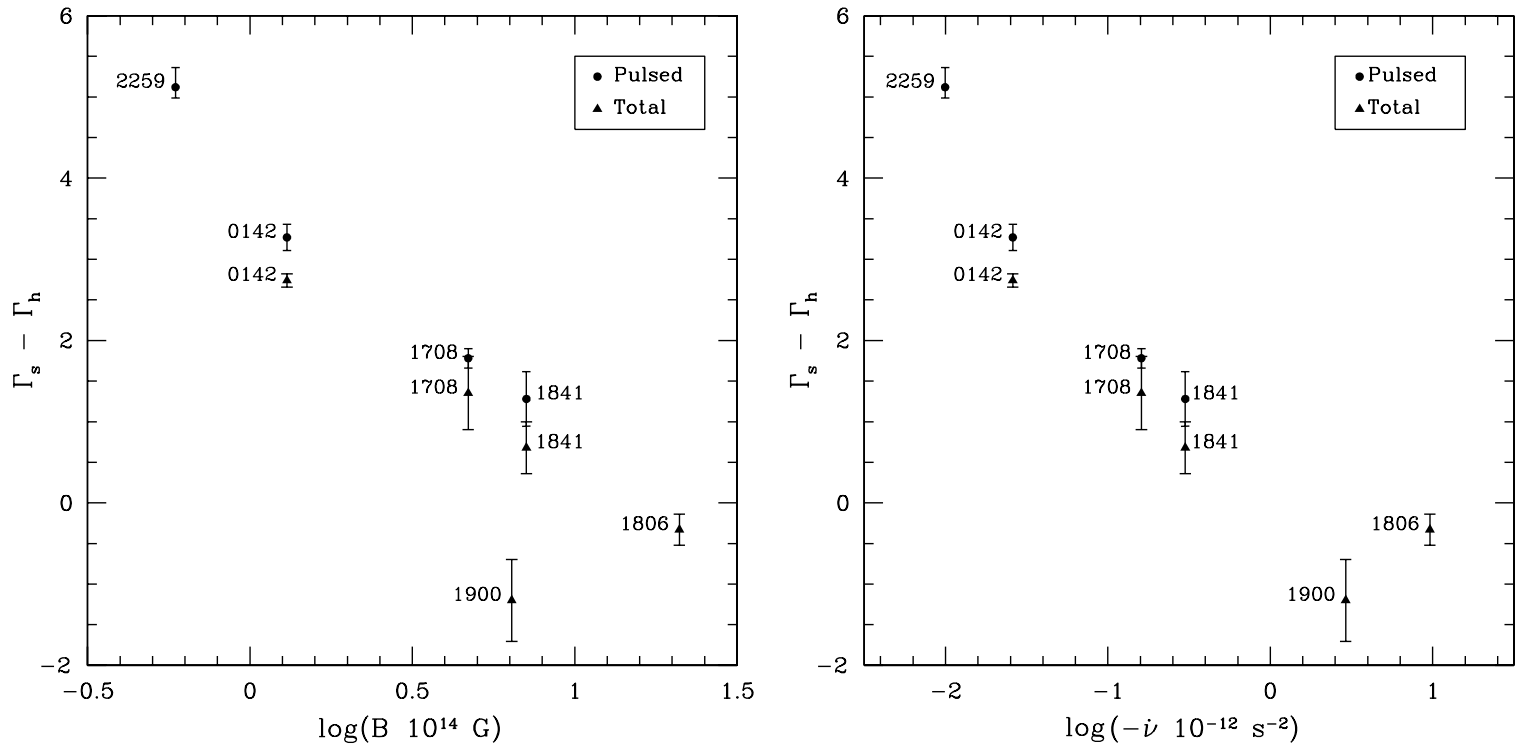

Figure 4. Left: spectral turnover, $\Gamma_{s}-\Gamma_{h}$, vs. $B$, for all magnetars for which both $\Gamma_{s}$ and $\Gamma_{h}$ are measured. Circles represent pulsed flux, and triangles represent total flux. See the text and Table 1 for data used and references. Right: same but vs. $\dot{v}$. Both: error bars represent $1 \sigma$ uncertainties. All known RPPs for which the quantity has been measured have $\Gamma_{s}-\Gamma_{h} \approx 0$, with $B$ in the range $1-10 \times 10^{12} \mathrm{G}$.

$\mathrm{X}$-ray telescopes given their sensitivity, barring another major outburst.

Figure 4 shows values of $\Gamma_{s}-\Gamma_{h}$, our parameterization of the degree of spectral turnover, plotted versus $B$ (left) and $\dot{v}$ (right). A trend is suggested by both plots, such that the lowest $B$ or $\dot{v}$ sources generally have the largest spectral turnover. Quantitatively, for the pulsed data, we find $r=-0.99$ for $N=4$ implying $p=0.014$ and $r=-0.98$ for $N=4$ implying $p=0.021$, for $\Gamma_{s}-\Gamma_{h}$ versus $B$ and $\dot{v}$, respectively. When considering total flux, these values change to $r=-0.90$ for $N=6$ implying $p=0.015$ and $r=-0.92$ for $N=6$ implying $p=0.0087$, for $\Gamma_{s}-\Gamma_{h}$ versus $B$ and $\dot{v}$, respectively, again including the (pulsed) data for 1 E $2259+586$, under the reasonable assumption that the difference between its total and pulsed flux spectral turnovers is small (as it is for the three sources for which both have been measured). Thus, these correlations are significant at the $\gtrsim 99 \%$ level. If the $\Gamma_{h}$ versus
$B$ trend holds and the above prediction for $\Gamma_{h}$ for 1E $1048.1-$ 5937 is correct, its spectral turnover would agree well with this second trend. The same obvious caution regarding the paucity of sources as in Figure 3 holds, however.

We also investigated whether there exists any correlation between hard X-ray luminosity and $B$, but found none. However, the luminosities of the sources in this band are generally poorly known, given relatively large $\Gamma_{h}$ and distance uncertainties. Thus some correlation may exist, but cannot presently be discerned.

\section{DISCUSSION}

We have revisited the correlation between soft X-ray band power-law spectral index and $\dot{v}$ and $B$ reported previously by MW01, and find it continues to hold, though with significant scatter. This stands in contrast to the absence of any apparent relationship between blackbody temperature and $B$ or $\dot{v}$. This 
supports the foundation for the "twisted magnetosphere" model of Thompson et al. (2002), as further developed by Thompson \& Beloborodov (2005) and Beloborodov \& Thompson (2007). The significant scatter in the still small measured population suggests in the context of this model that the inferred $B$ is only a rough estimate of the stellar dipolar magnetic field, and/or that other factors, such as geometry (both intrinsic and viewing) and higher-order magnetic multipoles, play physically important roles. With very recent discoveries of several new magnetars, for example, SGR 0501+4516 (Enoto et al. 2009) and SGR 0418+5729 (van der Horst et al. 2009), as well as new measurements of spin parameters for previously known objects, as, for example, for SGR 1627 - 41 (Esposito et al. 2009), it should be possible to further test the veracity of the correlation in the near future.

The possible correlations we have noted for the hard-band emission from magnetars may be telling us something new about the magnetar emission process. Next, we consider whether the published models have predicted the observed trends.

Heyl \& Hernquist (2005) suggested that magnetohydrodynamic fast modes produced by magnetars may break down to form electron/positron pairs in the magnetosphere. They suggested that nonthermal radiation associated with this fast-mode breakdown could account for the high-energy emission from magnetars. However, in this model, the spectrum of the radiation is fixed and is not expected to vary from source to source as has been observed. This difficulty, along with the now falsified (den Hartog et al. 2008b) prediction that the fast-mode breakdown radiation spectrum should extend above $1 \mathrm{MeV}$, argues against this model being relevant to explain the observed properties of the hard X-ray emission in magnetars.

Baring \& Harding (2007) explore resonant inverse-Compton upscattering of thermal emission from the surface, by relativistic electrons only a few stellar radii from the surface. This resonant scattering, at least in their preliminary analysis, is expected to yield a very flat spectrum, independent of $B$, unlike what is reported here. However, Baring \& Harding (2007) did not consider non-resonant scattering which they suggest may have a very different spectrum, although its relative contribution is as yet unclear. For the resonant scattering, although substantial spectral differences are expected for different scattering locales, as well as for more complicated field geometries than the assumed dipole, the flatness of the spectrum independent of $B$ seems robust. The correlations we report thus suggest that this process is not the dominant mechanism for producing the hard X-ray emission in magnetars.

Thompson et al. (2002), Thompson \& Beloborodov (2005), and Beloborodov \& Thompson (2007) argue that powerful magnetic currents in the magnetar magnetosphere are induced by sporadic starquakes that twist the external magnetic field. A self-induction electric field lifts particles from the surface, accelerates them, and yields avalanches of pair creation. This forms a corona close to the neutron star surface. Thompson \& Beloborodov (2005) and Beloborodov \& Thompson (2007) consider a model in which the hard X-rays are produced in the transition layer between the corona and thermal photosphere. In this model, the hard X-ray emission is a result of thermal bremsstrahlung with a temperature that scales approximately as $k T \propto B^{2 / 5}$, with $k T \simeq 100 \mathrm{keV}$ for reasonable parameters. However, we verified, using spectral simulations, that this scaling predicts the opposite of the correlation seen in Figure 3, because higher $k T$, expected for higher $B$, gets fit with a lower $\Gamma_{h}$ when fits with a simple power law, for $k T$ in the range
20-200 keV. Thus the observed correlation does not seem consistent with a thermal bremsstrahlung origin.

On the other hand, the observed correlation between the spectral index change and $B$ could be interpreted at least qualitatively in the framework of models in which magnetospheric currents play a role in the production of the high-energy emission as follows. In the magnetosphere, relativistic electrons can resonantly scatter surface thermal photons to form the nonthermal portion of the soft-band spectrum as discussed first by Thompson et al. (2002). Alternatively, electrons that do not have a chance to scatter will impact on the surface, heating it, and possibly creating a corona as described by Thompson \& Beloborodov (2005) and further explored by Beloborodov \& Thompson (2007) (but see Lyubarsky \& Eichler 2007). For higher $B$ sources, the scattering optical depth increases, rendering the soft index harder as described by Thompson et al. (2002), but leaving fewer electrons for heating the corona, and hence possibly resulting in the hard index being softer.

For energetic RPPs, the power-law spectrum seen below $10 \mathrm{keV}$ extends well beyond that energy, such that the spectral turnover $\Gamma_{s}-\Gamma_{h} \approx 0$ for practically all sources for which it has been measured. In a handful of cases, there is marginal evidence for a subtle change of shape of the hard spectrum, generally parameterized as a log-parabolic steepening above $10 \mathrm{keV}$ (e.g., Campana et al. 2008). Clearly if the trend in Figure 4 held for RPPs, one would expect huge turnovers, given their far lower $B$. This argues strongly that the mechanism for the production of hard X-rays in magnetars is different in RPPs, which was already suggested by the fact that the magnetar hard X-ray emission has luminosity far in excess of that available from rotational slow-down, in contrast to that from RPPs. $\Gamma_{h}$ for the usually rotation-powered PSR J1846 - 0258 (but see Gavriil et al. 2008), the highest- $B\left(4.9 \times 10^{13} \mathrm{G}\right)$ RPP for which $\Gamma_{h}$ has been measured (Kuiper \& Hermsen 2009), is 1.2, much larger than would be predicted by the trend in Figure 3, left $\left(\Gamma_{h}\right.$ vs. $B$ ) but smaller than what would be predicted in Figure 3, right $\left(\Gamma_{h}\right.$ vs. $\left.\dot{v}\right)$. Indeed, the same is true of practically all RPPs for which $\Gamma_{h}$ has been measured, further demonstrating a different hard-X-ray production mechanism.

We thank A. Beloborodov, D. Eichler, M. Livingstone, and C. Thompson for useful conversations. We thank Caltech for its hospitality and support via a Summer Undergraduate Research Fellowship (K.B.) and a Moore Scholarship (V.M.K.). V.M.K. receives additional support from NSERC, FQRNT, CIFAR, and holds a Canada Research Chair and the Lorne Trottier Chair in Astrophysics and Cosmology.

\section{REFERENCES}

Baring, M. G., \& Harding, A. K. 2007, Ap\&SS, 308, 109

Beloborodov, A. M., \& Thompson, C. 2007, ApJ, 657, 967

Campana, R., Mineo, T., de Rosa, A., Massaro, E., Dean, A. J., \& Bassani, L. 2008, MNRAS, 389, 691

Campana, S., Rea, N., Israel, G. L., Turolla, R., \& Zane, S. 2007, A\&A, 463, 1047

den Hartog, P. R., Hermsen, W., Kuiper, L., Vink, J., in't Zand, J. J. M., \& Collmar, W. 2006, A\&A, 451, 587

den Hartog, P. R., Kuiper, L., \& Hermsen, W. 2008a, A\&A, 489, 263

den Hartog, P. R., Kuiper, L., Hermsen, W., Kaspi, V. M., Dib, R., Knoedlesder, J., \& Gavriil, F. P. 2008b, A\&A, 489, 245

Enoto, T., et al. 2009, ApJ, 693, L122

Esposito, P., Mereghetti, S., Tiengo, A., Sidoli, L., Feroci, M., \& Woods, P. 2007, A\&A, 461, 605

Esposito, P., et al. 2009, MNRAS, 399, L44

Gavriil, F. P., Dib, R., \& Kaspi, V. M. 2009, ApJ, submitted (arXiv:0905.1256) 
Gavriil, F. P., Gonzalez, M. E., Gotthelf, E. V., Kaspi, V. M., Livingstone, M. A., \& Woods, P. M. 2008, Science, 319, 1802

Gelfand, J. D., \& Gaensler, B. M. 2007, ApJ, 667, 1111

Gotthelf, E. V., Halpern, J. P., Buxton, M., \& Bailyn, C. 2004, ApJ, 605, 368

Götz, D., Mereghetti, S., Tiengo, A., \& Esposito, P. 2006, A\&A, 449, L31

Heyl, J. S., \& Hernquist, L. 2005, ApJ, 618, 463

Israel, G. L., Campana, S., Dall'Osso, S., Muno, M. P., Cummings, J., Perna, R., \& Stella, L. 2007, ApJ, 664, 448

Kaspi, V. M., Gavriil, F. P., Woods, P. M., Jensen, J. B., Roberts, M. S. E., \& Chakrabarty, D. 2003, ApJ, 588, L93

Kaspi, V. M., \& McLaughlin, M. A. 2005, ApJ, 618, L41

Kuiper, L., \& Hermsen, W. 2009, A\&A, 501, 1031

Kuiper, L., Hermsen, W., den Hartog, P., \& Collmar, W. 2006, ApJ, 645, 556

Kuiper, L., Hermsen, W., \& Mendez, M. 2004, ApJ, 613, 1173

Kulkarni, S. R., Kaplan, D. L., Marshall, H. L., Frail, D. A., Murakami, T., \& Yonetoku, D. 2003, ApJ, 585, 948

Lyubarsky, Y., \& Eichler, D. 2007, arXiv:0706.3578

Lyutikov, M., \& Gavriil, F. P. 2006, MNRAS, 368, 690

Marsden, D., \& White, N. E. 2001, ApJ, 551, L155

McGarry, M. B., Gaensler, B. M., Ransom, S. M., Kaspi, V. M., \& Veljkovik, S. 2005, ApJ, 627, L137

McLaughlin, M. A., et al. 2003, ApJ, 591, L135

Mereghetti, S., Götz, D., Mirabel, I. F., \& Hurley, K. 2005, A\&A, 433, L9

Mereghetti, S., et al. 2006, ApJ, 653, 1423

Molkov, S. V., Cherepashchuk, A. M., Lutovinov, A. A., Revnivtsev, M. G., Postnov, K. A., \& Sunyaev, R. A. 2004, Sov. Astron. Lett., 30, 534
Morii, M., Sato, R., Kataoka, J., \& Kawai, N. 2003, PASJ, 55, L45

Nobili, L., Turolla, R., \& Zane, S. 2008, MNRAS, 386, 1527

Özel, F., \& Guver, T. 2007, ApJ, 659, L141

Patel, S. K., et al. 2001, ApJ, 563, L45

Pivovaroff, M., Kaspi, V. M., \& Camilo, F. 2000, ApJ, 535, 379

Rea, N., Oosterbroek, T., Zane, S., Turolla, R., Méndez, M., Israel, G. L., Stella, L., \& Haberl, F. 2005, MNRAS, 361, 710

Rea, N., Turolla, R., Zane, S., Tramacere, A., Stella, L., Israel, G. L., \& Campana, R. 2007a, ApJ, 661, L65

Rea, N., et al. 2007b, Ap\&SS, 308, 505

Tam, C. R., Gavriil, F. P., Dib, R., Kaspi, V. M., Woods, P. M., \& Bassa, C. 2008, ApJ, 677, 503

Thompson, C., \& Beloborodov, A. M. 2005, ApJ, 634, 565

Thompson, C., Lyutikov, M., \& Kulkarni, S. R. 2002, ApJ, 574, 332

Tiengo, A., Mereghetti, S., Turolla, R., Zane, S., Rea, N., Stella, L., \& Israel, G. L. 2005, A\&A, 437, 997

van der Horst, A. J., et al. 2009, ATEL, 2077

White, N. E., Angelini, L., Ebisawa, K., Tanaka, Y., \& Ghosh, P. 1996, ApJ, 463, L83

Woods, P. M., Kaspi, V. M., Gavriil, F. P., \& Airhart, C. 2009, ApJ, submitted Woods, P. M., Kouveliotou, C., Finger, M. H., Göğüş, E., Wilson, C. A., Patel, S. K., Hurley, K., \& Swank, J. H. 2007, ApJ, 654, 470

Woods, P. M., et al. 2004, ApJ, 605, 378

Zane, S., Rea, N., Turolla, R., \& Nobili, L. 2009, MNRAS, 398, 1403 